\title{
DEVELOPMENT OF NON-REFLECTING BOUNDARY CONDITION FOR APPLICATION IN 3D COMPUTATIONAL FLUID DYNAMIC CODES
}

\author{
A. J. Torregrosa, P. Fajardo*, A. Gil and R. Navarro \\ CMT - Motores Térmicos, Universitat Politècnica de València \\ Camino de Vera S/N, 46022 Valencia, Spain \\ *E-Mail: pabfape@mot.upv.es (Corresponding Author)
}

\begin{abstract}
Numerical computations are commonly used for better understanding the unsteady processes in internal combustion engine components and their acoustic behavior. The acoustic characterization of a system requires that reflections from duct terminations are avoided, which is achieved either by using highly dissipative terminations or, when an impulsive excitation is used, by placing long ducts between the system under study and the duct ends. In the latter case, the simulation of such a procedure would require a large computational domain with the associated high computational cost, unless non-reflecting boundary conditions are used. In this paper, first the different non-reflecting boundary conditions available in ANSYS-FLUENT are evaluated. Then, the development and implementation of an anechoic termination in a 3D-CFD code is presented. The performance of the new implementation is first validated in the classic Sod's shock tube problem, and then checked against numerical and experimental results of the flow and acoustic fields in automotive exhaust mufflers. The results obtained compare favorably with those from the conventional CFD approach and experiments, while the computational cost is significantly reduced.
\end{abstract}

Keywords: non-reflecting boundary condition, CFD simulation, method of characteristics, anechoic end, acoustic response, exhaust muffler

\section{INTRODUCTION}

In the last few decades, increasingly restrictive legislation and the need to reduce development costs of internal combustion engines (ICE) have led to considerable efforts seeking the improvement of the fluid-dynamic and acoustic behavior of the different components of an ICE. In particular, the use of numerical simulations for muffler evaluation and design has become common practice.

Among the different techniques available, those most used are based upon a one-dimensional (plane-wave) approach, due to their extremely low computational cost. Such codes are particularly well suited for the computation of wave action in the ducts of intake and exhaust systems and its effects on ICE performance and noise emissions. However, these do not deal with the actual geometry and rely upon quasi-steady assumptions. In cases in which the geometry is relatively simple, one-dimensional (1D) codes provide good resolution even in compressible unsteady flows. However, for more realistic geometries, it is imperative to resort to the numerical solution of three-dimensional (3D) wave propagation.
3D analyses are usually achieved in the linear regime, by means of the Finite Element Method (FEM), as described in Patil et al. (1996), or the Boundary Element Method (BEM), in order to obtain magnitudes in the frequency domain, such as the transmission loss. These methods have been extensively used for design purposes, even if they present some limitations when handling anisotropy or when describing the coupling between the mean flow and the fluctuations (Tsuji et al., 2002).

A further step is the use of full 3D-CFD simulations in order to describe the unsteady flow behavior of a system. In these codes, the full flow equations are solved in the time domain, and then the information can be transformed into the frequency domain for its analysis. The main advantage of these methods is that, since turbulence is modeled, its dissipative effects are also taken into account. Some previous work by Broatch et al. (2005) and Montenegro and Onorati (2009) showed the potential benefits of CFD, with good agreement with experiments when modeling both mean and pulsating flow in actual exhaust mufflers. The main problem when using 3D-CFD codes is the CPU time consumed during the computations given that an extension of the computational domain, by means of long ducts, is

Received: 15 Oct. 2011; Revised: 29 Feb. 2012; Accepted: 16 Apr. 2012 
required in order to capture isolated pressure signals upstream and downstream of the system under study in order to compute its acoustic response. Onorati et al. (2006) and Galindo et al. (2011) solved this problem by performing coupled 1D-3D CFD computations, which allowed for the use of 1D codes in some regions of the domain while still conserving a good geometrical resolution where required.

In this frame of ideas, the development of nonreflecting boundary conditions (NRBCs) for their use in 3D-CFD acoustic computations appears to be a suitable alternative. With these boundary conditions the use of long ducts to capture the acoustic response of the system analyzed is not necessary, so that the CPU-time required is considerably reduced. This methodology has similar advantages to those of using 1D models to simulate the flow in the ducts, and the full 3DCFD calculations are performed where required to take into account the singularity of complex geometries. Of course, NRBCs are used in a wide range of different applications (Givoli, 2004; Lindquist et al., 2010).

The literature on the implementation of NRBCs usually groups the boundary conditions into three categories. The first one comprises the methods based on a decomposition into Fourier modes (Atassi, 2004; Hagstrom and Hariharan, 1988; Dorodnicyn, 2010). In the second category the conservation equations are extrapolated in order to obtain asymptotic solutions (Tam, 1995; Bogey and Bailly, 2002; Dea et al., 2009). Methods that follow the classical absorbing boundary condition developed by Engquist and Majda (1977) fall into this category. Finally, the third category includes methods based on the Method of Characteristics (MoC) (Thompson, 1987 and 1990; Liu and Vasilyev, 2010), which is also the basis of the work presented here.

While some NRBCs have been developed for internal flow problems (Atassi, 2004; Morínigo and Salvá, 2010), most of the existing NRBCs were conceived for their application to external flow problems (Hagstrom and Hariharan, 1988), and cannot be used in a straightforward manner in internal flow simulations. CFD commercial codes usually include some boundary conditions claimed to be non-reflecting. However, as will be shown later, the performance of those included in ANSYS-FLUENT is not the most appropriate for the problem under consideration. The objective of this paper is to develop and implement a new non-reflecting boundary condition for its use in ANSYS-FLUENT. The main difficulty involved arises from the dependence of the implementation on the flow variables arriving at the boundary.

The paper is organized as follows: First, the theoretical definition of NRBCs is described. Then, the non-reflecting boundary conditions available in ANSYS-FLUENT are analyzed, showing their limitations when a nonhomentropic problem is considered. In order to overcome those limitations, the issues associated with the inclusion of a new boundary condition in a commercial 3D-CFD code are addressed, and a first validation of the procedure for an existing NRBC is shown. An improved NRBC is proposed and implemented in ANSYS-FLUENT. In the last section, this boundary condition is applied to reproduce the acoustic behavior of a real 3D muffler. The results obtained, comprising both the pressure evolution in the time domain and the transmission loss in the frequency domain, are evaluated by comparison with experimental measurements and with the results from conventional full 3D CFD calculations indicating, in view of the quality of the results and the substantial reduction achieved in the computation time, the suitability of the proposed methodology for design and evaluation purposes.

\section{NON-REFLECTING BOUNDARY CONDITIONS}

As stated in the previous section, an NRBC renders unnecessary the use of a long duct in order to avoid the reflection of pressure waves at the boundary, and therefore it may be expected to work as an infinite duct. The implementation of such a boundary condition in a 3D-CFD code will reduce considerably the computation time. This reduction gains importance if the main interest of the problem lies in a particular section of the domain and the rest of the elements are geometrically simple, such as ducts. A good example of this kind of application could be the simulation of an impulse test rig (with about $20 \mathrm{~m}$ of ducts both at the inlet and outlet) (Payri et al., 2000) or a turbocharger test rig (Luján et al., 2002). The reduction in computation time achieved when the ducts are removed is related to two different aspects. First, the number of cells in the domain is reduced and hence the CPU time per time-step. Secondly, and even more important, one may save all the time required for the information to propagate along the ducts and reach the system under study.

Once the flow variables are known, it is possible and convenient to decompose them in order to identify separately the information propagating in 
both forward $\left(p_{f}\right)$ and backward $\left(p_{b}\right)$ directions. Such a decomposition might be performed by applying Eq. (1), as shown by Payri et al. (1995).

$$
\begin{aligned}
& p_{f}=p_{0}\left[\frac{1}{2}\left(1+\left(\frac{p}{p_{0}}\right)^{\frac{\gamma-1}{2 \gamma}}\left(1+\frac{\gamma-1}{2} \frac{u}{a}\right)\right)\right]^{\frac{2 \gamma}{\gamma-1}} \\
& p_{b}=p_{0}\left[\frac{1}{2}\left(1+\left(\frac{p}{p_{0}}\right)^{\frac{\gamma-1}{2 \gamma}}\left(1-\frac{\gamma-1}{2} \frac{u}{a}\right)\right]^{\frac{2 \gamma}{\gamma-1}}\right.
\end{aligned}
$$

Then it is possible, according to Mucklow and Wilson (1955), to recover the pressure from its forward and backward components as follows:

$$
\left(\frac{p}{p_{0}}\right)^{\frac{\gamma-1}{2 \gamma}}=\left(\frac{p_{f}}{p_{0}}\right)^{\frac{\gamma-1}{2 \gamma}}+\left(\frac{p_{b}}{p_{0}}\right)^{\frac{\gamma-1}{2 \gamma}}-1 \text {. }
$$

This decomposition will be referred to frequently in the rest of the discussion. Returning to the NRBCs, two different kinds can be defined:

i. The incident pressure boundary condition, which is an inlet condition in which the flow variables at the boundary are not known, but instead information is available on the pressure component coming from upstream of the boundary $\left(p_{f}\right)$. Therefore, computing the flow field is required in order to recover the pressure boundary condition by means of Eq. (2).

ii. An anechoic end, which is an outlet boundary condition in which information does not propagate upstream of the boundary.

In view of Eq. (1) the previous definition of an anechoic end may suggest setting $p=p_{f}$ (whence $p_{b}=p_{0}$ ) at the boundary. However, it will be shown later that this is incorrect. It is remarkable that an anechoic end is physically meaningful only when the flow goes out of the domain. The main contributions of this paper are related with the anechoic condition, but the conclusions may be easily extended to the incident pressure boundary condition.

\subsection{Assessment of NRBCs available in ANSYS-FLUENT}

Three different kinds of NRBC are currently available in ANSYS-FLUENT (ANSYS Inc., 2009):
1. Turbo-specific non-reflecting boundary condition, which is not considered in this paper as it can only be used for steadystate calculations with the density-based solver and structured meshes.

2. General non-reflecting boundary condition, which is applicable only with the density-based solver but can be used in both steady and unsteady simulations without any geometric restrictions. This boundary condition is based on the characteristic analysis developed by Thompson (1987 and 1990), after which the Linear Relaxation Method (LRM) is used to determine the value of the incoming wave amplitude, as shown by Poinsot and Lele (1992) and Selle et al. (2004). There are two versions of this boundary condition:

a. Pressure at infinity, in which the pressure at the boundary is relaxed toward the imposed pressure at infinity.

b. Average boundary pressure, in which the average pressure on the boundary is forced to approach the exit pressure value.

3. Pressure far-field boundary condition, which uses Riemann invariants to determine the flow variables at the boundaries and has no constraints regarding solver or mesh type.

In order to check the validity of the NRBCs available in ANSYS-FLUENT, they were checked against the well-known Sod's shock tube. The solver used for these simulations was a density based solver with an explicit time discretization. Sod's problem (Sod, 1978) is a shock tube problem commonly used to test the accuracy of computational fluid codes (Wang and Xu, 2007; Elfaghi et al., 2010; Qamar et al., 2010). The interest of this problem lies in the fact that an analytical solution is available when viscosity is neglected. The test consists in the transient evolution in a shock tube, divided initially by means of a membrane into two separate regions which are set to different pressures and temperatures (for the sake of simplicity, here the same temperature was initially set in both regions). At time zero, the membrane is removed and the flow inside the tube starts to evolve. Sod's shock tube is quite a challenging problem, since the boundary has to deal with changes in entropy, 
which are not present in homentropic flow but are likely to be found in the conditions expected in an ICE. In the case studied, a tube of $1 \mathrm{~m}$ length was simulated with an outlet NRBC, as can be seen in Fig. 1. The membrane was located at $x=0.8 \mathrm{~m}$ and fluid-flow variables were registered at a measuring section at $x=0.9 \mathrm{~m}$. The behavior of the NRBCs previously described was then checked.

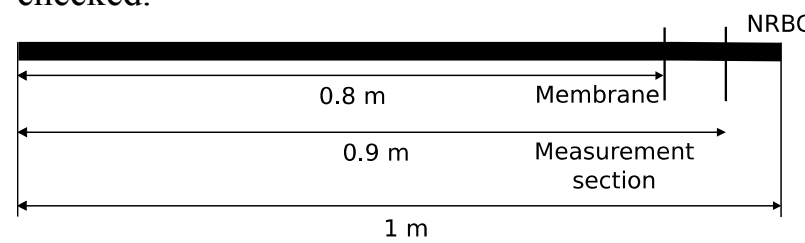

Fig. 1 Computational domain of Sod's problem used to validate the NRBCs.

As indicated above, an anechoic end is expected to behave as an infinite duct. Therefore, the results obtained in the simulations were compared with those of simulating a duct sufficiently long so as to avoid any possible reflection at the boundary, thus behaving locally as an infinite duct. In Fig. 2, the pressure decomposition and the Riemann variables at the measuring section of the long duct are shown. At $0.22 \times 10^{-3} \mathrm{~s}$, a $\lambda$ wave arrives and pressure rises. Then, at $0.69 \times 10^{-3} \mathrm{~s}$, the entropy discontinuity modifies $p_{f}$ and $p_{b}$ without any change in the static

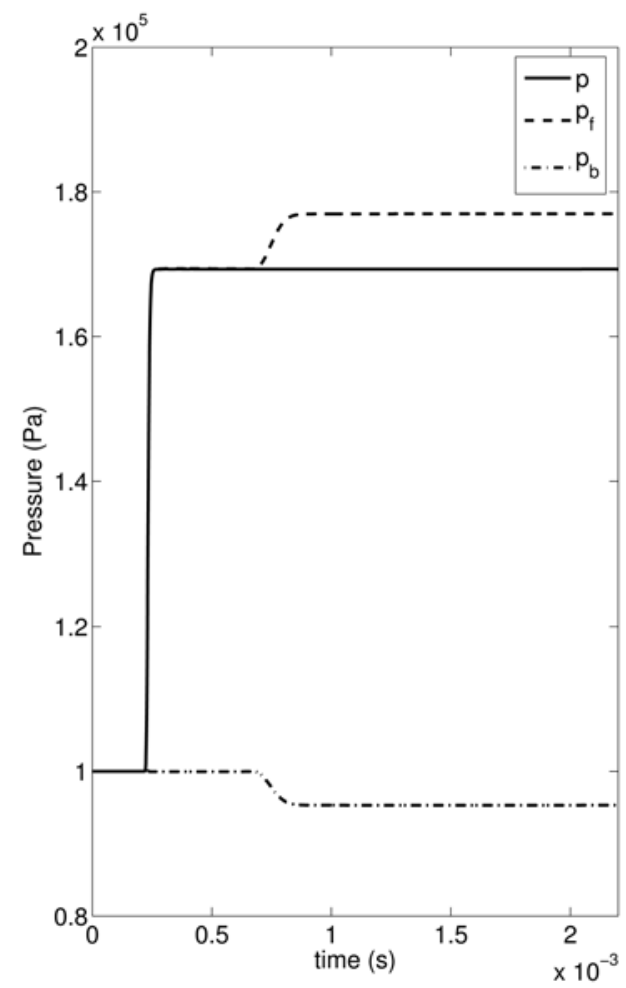

pressure. The other Riemann variables $(\lambda$ and $\beta$ ) are also modified due to the entropy variation. After that, everything remains unchanged.

Performance of the different NRBCs is presented in Fig. 3. It appears that none of them can be regarded as a proper anechoic boundary condition, since they do not represent satisfactorily the behavior of a long duct. Both general nonreflecting boundary conditions produce a spurious $\beta$ rise at $0.77 \times 10^{-3} \mathrm{~s}$ when the $\lambda$-wave reaches the boundary. As a consequence, a non-physical pressure jump is produced. Moreover, the pressure at infinity version, even if it is considered as more adequate for unsteady flow simulations than its counterpart (ANSYS Inc., 2009), actually exhibits some noise after the spurious rise in pressure. Finally, the pressure far field version does not produce any reflection upon arrival of a $\lambda$-wave. However, it produces an unreal pressure rise at $1.87 \times 10^{-3} \mathrm{~s}$, due to the spurious $\beta$ variation originated when the entropy discontinuity reaches the boundary, as shown in Fig. 3. The error underlying this boundary condition is that the backward invariant, i.e., $\beta$, is regarded as constant, whereas in Fig. 2 it can be seen that it is modified due to variations in the entropy level. This consideration is taken into account in section 3 below for the development of the proposed anechoic boundary condition.

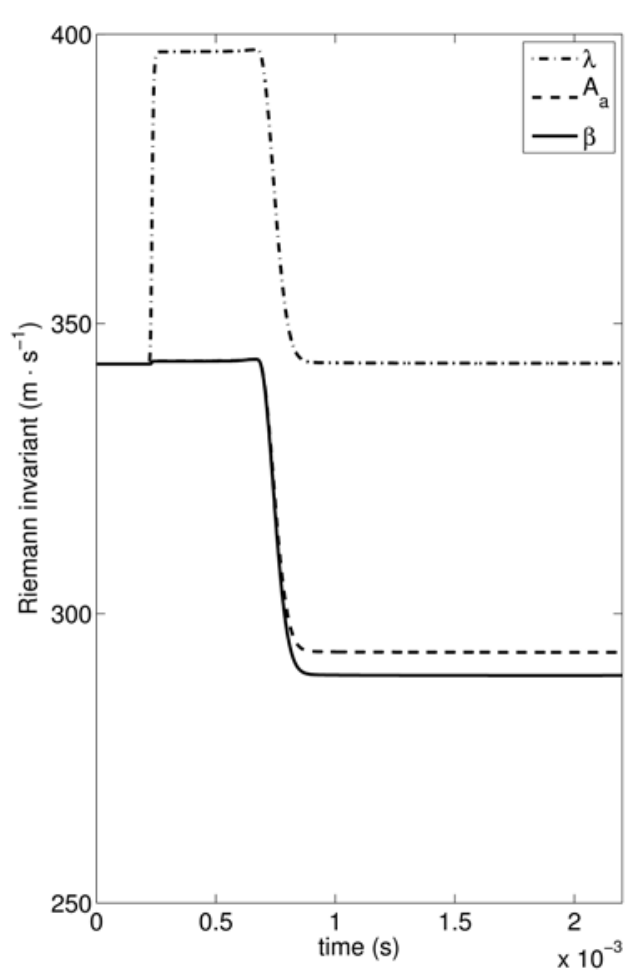

Fig. 2 Values of flow variables: pressure and decomposed pressure (left) and Rieman variables (right) in ideal infinite tube. 


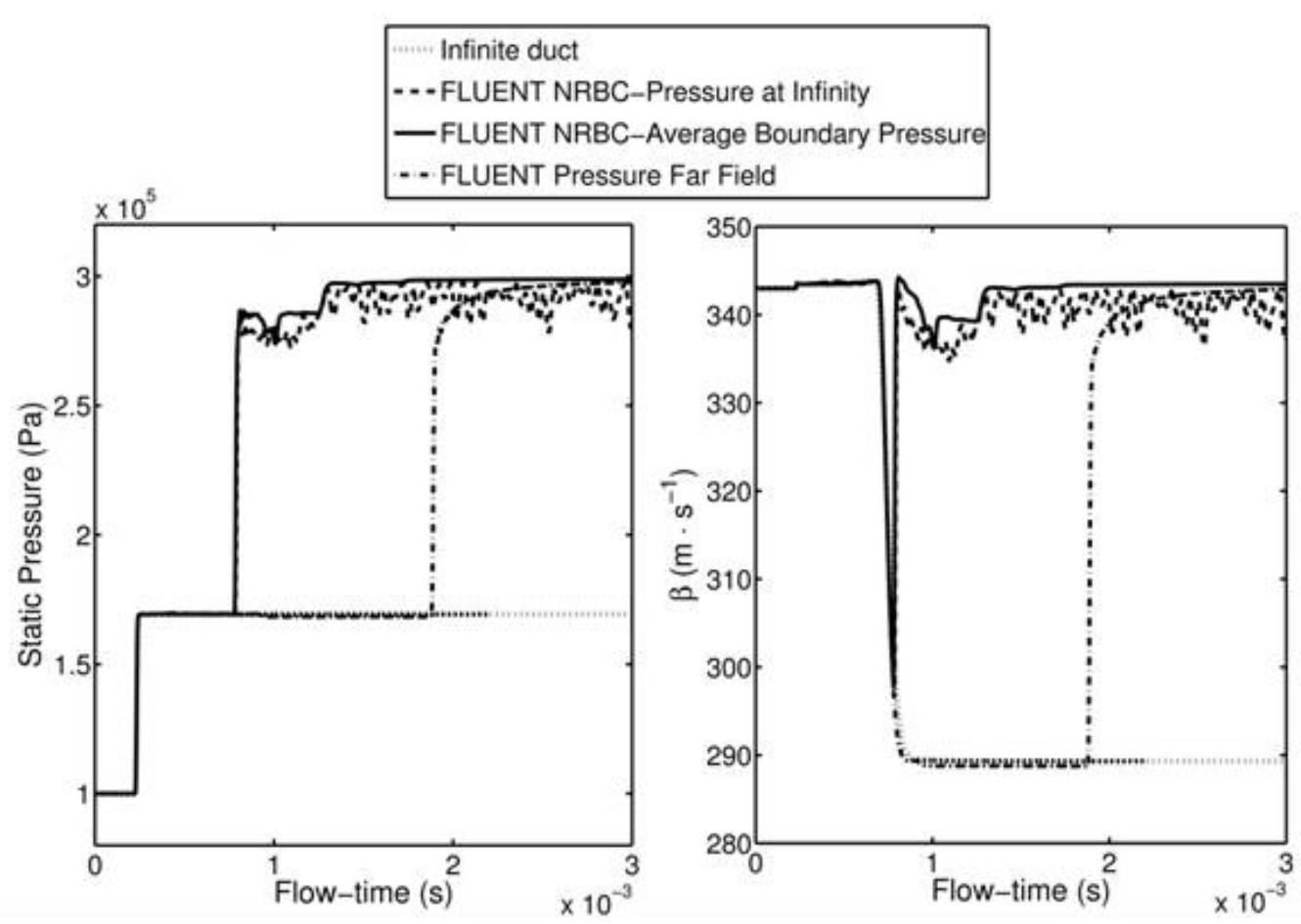

Fig. 3 Values of flow variables: pressure (left) and $\beta$ (right) in short tube using different NRBCs implemented in ANSYS-FLUENT and in ideal infinite tube.

\subsection{Implementing boundary condition in CFD commercial code}

One of the main drawbacks when dealing with commercial CFD codes is the lack of access to the fluid variables in the entire domain, as they are necessary to compute the proper values at the boundary. The methodology used in the following to account for information exchange across the boundary is based on that described in Galindo et al. (2011), where the Method of Characteristics (MoC) was applied in order to perform a coupled 1D-3D CFD simulation. The $\mathrm{MoC}$ is a mathematical technique for solving hyperbolic systems of partial differential equations (PDEs), in which the system of PDEs is reduced to a family of ordinary differential equations (ODEs), thus enabling one to obtain a solution from some initial data. The MoC establishes that there are certain flow variables, known as Riemann invariants $\left(\lambda, \beta\right.$, and $\left.A_{a}\right)$, which remain constant along certain lines, known as characteristic lines. In the case of homentropic flow the Riemann invariants are given by

$$
\begin{aligned}
& \lambda=a+\frac{\gamma-1}{2} u, \quad \beta=a-\frac{\gamma-1}{2} u \\
& A_{a}=a\left(\frac{p_{0}}{p}\right)^{\frac{\gamma-1}{2 \gamma}}
\end{aligned}
$$

The approach used in the present study is similar, but there is no need to perform the coupled simulation, since the problem is decoupled, i.e. different elements of the domain can be calculated independently, given that the direction of the flow does not change and that the incident conditions are known. In order to check the viability of the implementation of an NRBC, the anechoic end implemented in OpenWAM was considered. This boundary condition is based on the developments of Payri et al. (1995), which lead to the condition $\beta=A_{a}$ at the boundary. Once the values of the flow variables are obtained for the domain, it is possible to compute the Riemann invariants $\lambda$ and $A_{a}$. The value of $\beta$ is then obtained from the application of the non-reflecting condition, as shown by Benson et al. (1964).

The results obtained from such a procedure are shown in Fig. 4, where it is clear that the relevant information has been successfully transferred from and to the computational domain. Nevertheless, a certain numerical error is found (marked with an A in Fig. 4), which might be attributed to the discretization, as suggested by Galindo et al. (2011). However, it is interesting to remark that this error vanishes after a few timesteps and therefore it only affects the solution momentarily. Comparing the result computed with this anechoic condition, plotted in Fig. 4, with the expected result presented in Fig. 2, it is possible to observe that this boundary condition does not truthfully represent an anechoic 
condition, since a pressure wave is reflected from the boundary. This can be seen in the changes that appear in the curves representing the reflected pressure, $p_{b}$ and $\beta$, which follow from setting $\beta=A_{a}$ at the boundary (mark B in Fig. 4).

\section{PROPOSAL OF NEW NON- REFLECTING BOUNDARY CONDITION FOR NON-HOMENTROPIC FLOW}

\subsection{Modifications for non-homentropic flow}

For each Riemann invariant to remain constant along its characteristic line, the flow must be assumed to be not only isentropic (i.e. inviscid and with negligible heat transfer) but also homentropic. If these assumptions do not hold, then the value of the Riemann invariants is not the same at two different points along their respective characteristic line and, therefore, they are referred to as Riemann variables instead of Riemann invariants. The corresponding expressions can be modified to account for entropy non-uniformity in the flow-field, and for the existence of wall friction or heat transfer through the walls. For instance, the variation of $\beta$ between two points of a characteristic line is provided by the following modifiers, which take into account friction $(f)$, heat transfer $(Q)$ and entropy variation $(S)$, respectively:

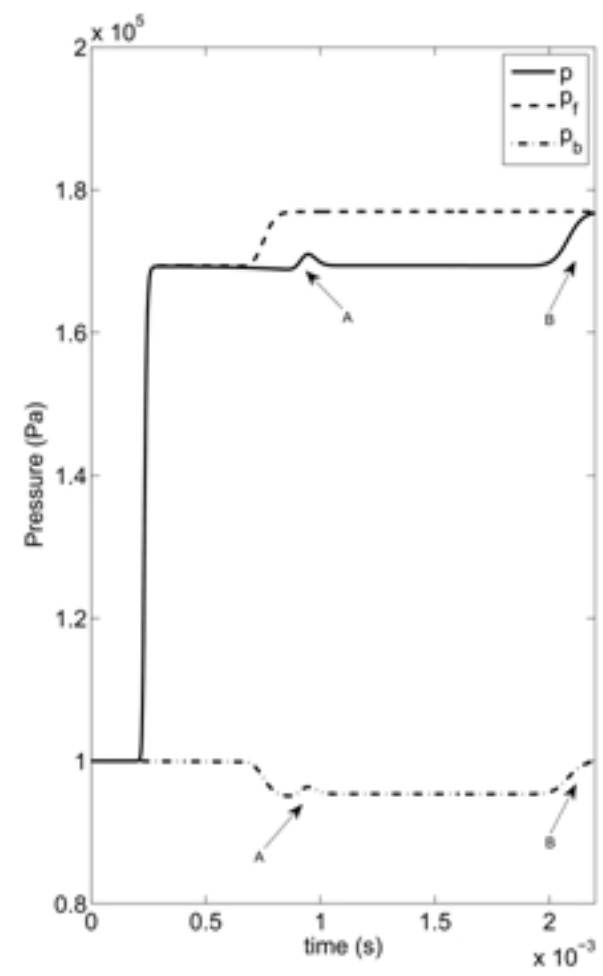

$$
\begin{aligned}
\left.\beta\right|_{2} & =\left.\beta\right|_{1}+\Delta \beta \\
& =a-\frac{\gamma-1}{2} u+\left.\Delta \beta\right|_{f}+\left.\Delta \beta\right|_{Q}+\left.\Delta \beta\right|_{S}
\end{aligned}
$$

where

$$
\begin{aligned}
\left.\Delta \beta\right|_{f} & =\left.\int_{1}^{2} \mathrm{~d} \beta\right|_{f}= \\
& =-\int_{1}^{2}(\gamma-1)\left[1-(\gamma-1) \frac{u}{a}\right] \frac{f}{D} u^{2} \frac{u}{|u|} \mathrm{d} t \\
\left.\Delta \beta\right|_{Q} & =\left.\int_{1}^{2} \mathrm{~d} \beta\right|_{Q}=\int_{1}^{2} \frac{(\gamma-1)^{2}}{2} q \frac{1}{a} \mathrm{~d} t \\
\left.\Delta \beta\right|_{S} & =\left.\int_{1}^{2} \mathrm{~d} \beta\right|_{S}=\int_{1}^{2} a \frac{\mathrm{d} A_{a}}{A_{a}} .
\end{aligned}
$$

Similar expressions may be obtained for the modifiers of the other two Riemann variables, as can be seen in Benson (1982). Particularly interesting in the case under consideration is the modification of the Riemann variables due to a change in the entropy of the flow along a characteristic line. The expression which allows one to compute such a variation may be discretized as:

$$
\left.\Delta \beta\right|_{S_{1 \rightarrow 2}}=\int_{1}^{2} a \frac{\mathrm{d} A_{a}}{A_{a}} \approx \frac{a_{1}+a_{2}}{2} \ln \left(\frac{A_{a_{2}}}{A_{a_{1}}}\right)
$$

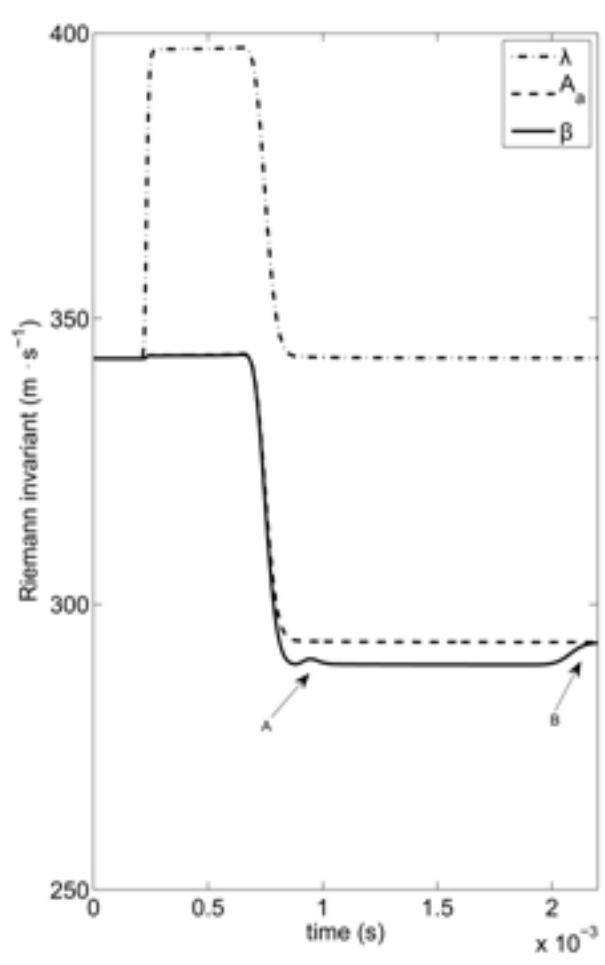

Fig. 4 Values of flow variables: pressure and pressure components (left) and Riemann variables (right) using former implementation of anechoic end, $\beta=A_{a}$. 


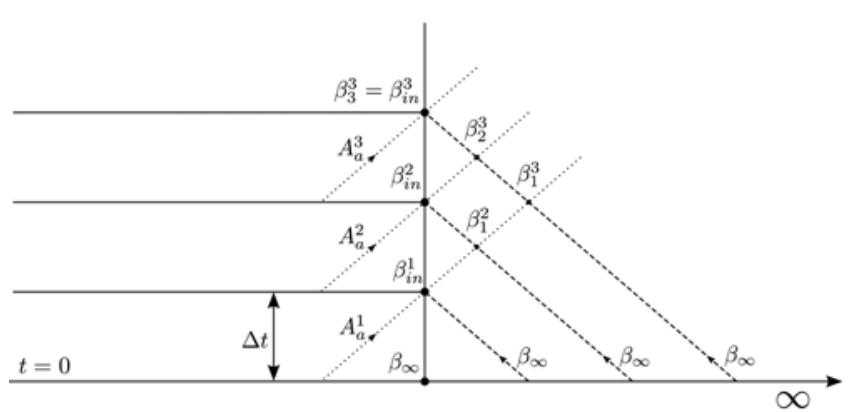

Fig.5 Modification of $\beta$ values due to changes of entropy occurring at crossing points of two characteristic lines.

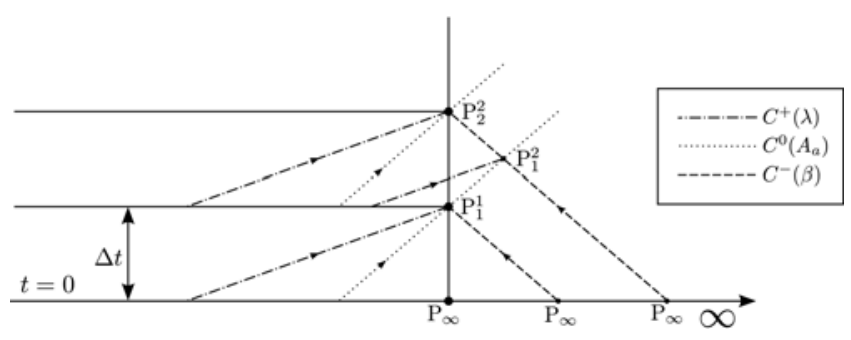

Fig.6 Schematic representation of characteristic lines at anechoic end. The points $P_{j}^{N}$ are defined following the criteria used in Eq.(7).

\subsection{Implementation of anechoic condition}

As previously stated, an anechoic end is expected to behave as an infinite duct. In terms of the MoC, such an infinite duct would always send back the same value of $\beta_{\text {out }}$, corresponding to the initial value of $\beta$ at the boundary, which hereinafter will be denoted as $\beta_{\infty}$. Thus, in the homentropic case one must keep the value of $\beta_{\text {in }}$ constant and equal to its value at the start of the simulation, i.e. $\beta_{\infty}$. Should a change of the entropy level happen, the actual value of $\beta_{\text {in }}$ would be no longer $\beta_{\infty}$. In this case, $\beta_{\text {in }}$ would be obtained by adding to $\beta_{\infty}$ the modifiers associated with the variation of the entropy level, corresponding to Eq. (5).

Therefore, the value of $\beta$ along the characteristic line $\left(C^{-}\right)$will be modified every time a different entropy level reaches this line. It is interesting to note that entropy levels are transported along the $\left(C^{0}\right)$ characteristic lines and thus the changes of entropy occur at the crossing points of the two characteristic lines (see Fig. 5). The corresponding changes in $\beta$ can be computed by applying Eqs. (4), (5) and (6). In this way, the value of $\beta$ at the $C$ characteristic line that will arrive at the boundary condition at the $N$-th time-step, just after crossing the $C^{0}$ characteristic line that passed through the boundary condition at the $j$-th time-step, is given by:
$\beta_{j}^{N}=\beta_{\infty}+\left.\sum_{i=1}^{j} \Delta \beta\right|_{s_{i-\rightarrow i}} ^{N}$

where subscript $i$ represents the time-step at which the different $C^{0}$ characteristic lines crossed the boundary condition. This can be seen in Fig. 5, where the characteristic lines have been represented as straight and parallel lines for the sake of simplicity. It is important to remark that $\beta_{N}^{N}$, which will be referred to as $\beta_{i n}^{N}$, corresponds to the value of $\beta$ that arrives at the boundary condition at the $N$-th time-step. For $i=0$, the values of the Riemann variables are those computed at the boundary condition at $t=0$, which are marked with subscript $\infty$.

Eq. (7) represents all the evolution along a characteristic line, from the downstream infinity to the boundary condition, so that it would be necessary in principle to compute the flow in the whole duct. Nevertheless, if the first time-steps are examined carefully (Fig.6), an approximate procedure can be devised. This is described next.

Applying Eq. (7) to the first time step $(N=1)$ and discretizing the modifiers according to Eq. (6), one gets

$\beta_{i n}^{1}=\beta_{1}^{1}=\beta_{\infty}+\frac{a_{1}^{1}+a_{\infty}}{2} \ln \frac{A_{a}^{1}}{A_{a}^{\infty}}$.

Now, since $a_{1}^{1}=\left(\lambda_{1}^{1}+\beta_{1}^{1}\right) / 2$, the following condition is readily obtained:

$$
\beta_{i n}^{1}=\left[\beta_{\infty}+\frac{a_{\infty}}{2} \ln \frac{A_{a}^{1}}{A_{a}^{\infty}}+\frac{\lambda_{1}^{1}}{4} \ln \frac{A_{a}^{1}}{A_{a}^{\infty}}\right]\left[1-\frac{1}{4} \ln \frac{A_{a}^{1}}{A_{a}^{\infty}}\right]^{-1}
$$

If the same reasoning is applied to $\lambda_{\text {in }}^{1}$, one obtains a linear system of two equations that allows one to compute $\beta_{i n}^{1}$ and $\lambda_{i n}^{1}$. In the same way, for the second time step, it is possible to show that

$\beta_{1}^{2}=\left[\beta_{\infty}+\frac{a_{\infty}}{2} \ln \frac{A_{a}^{1}}{A_{a}^{\infty}}+\frac{\lambda_{1}^{2}}{4} \ln \frac{A_{a}^{1}}{A_{a}^{\infty}}\right]\left[1-\frac{1}{4} \ln \frac{A_{a}^{1}}{A_{a}^{\infty}}\right]^{-1}$

and that

$\beta_{2}^{2}=\left[\beta_{1}^{2}+\frac{a_{1}^{2}}{2} \ln \frac{A_{a}^{2}}{A_{a}^{1}}+\frac{\lambda_{2}^{2}}{4} \ln \frac{A_{a}^{2}}{A_{a}^{1}}\right]\left[1-\frac{1}{4} \ln \frac{A_{a}^{2}}{A_{a}^{1}}\right]^{-1}$

In order to obtain an equation for $\beta_{2}^{2}$ involving only the variables arriving at the boundary condition, it may be assumed that 


$$
\begin{aligned}
& \lambda_{1}^{2} \simeq \lambda_{1}^{1} \\
& a_{1}^{2} \simeq a_{1}^{1}
\end{aligned}
$$

which, taking into account Eqs. (9), (10) and (11), gives the following approximation for $\beta_{2}^{2}$ :

$\tilde{\beta}_{2}^{2}=\left[\beta_{1}^{1}+\frac{a_{1}^{1}}{2} \ln \frac{A_{a}^{2}}{A_{a}^{1}}+\frac{\lambda_{2}^{2}}{4} \ln \frac{A_{a}^{2}}{A_{a}^{1}}\right]\left[1-\frac{1}{4} \ln \frac{A_{a}^{2}}{A_{a}^{1}}\right]^{-1}$

Recalling Eqs. (10), (11) and (13), the relative error of this approximation can be written as:

$$
\begin{aligned}
\frac{\beta_{2}^{2}-\tilde{\beta}_{2}^{2}}{\beta_{2}^{2}} & =\left[\left(\lambda_{1}^{2}-\lambda_{1}^{1}\right) \ln \frac{A_{a}^{1}}{A_{a}^{\infty}}\left(4-\ln \frac{A_{a}^{1}}{A_{a}^{\infty}}\right)^{-1}+\right. \\
& \left.+\frac{a_{1}^{2}-a_{1}^{1}}{2} \ln \frac{A_{a}^{2}}{A_{a}^{1}}\right] \\
& \times\left[\beta_{1}^{2}+\frac{a_{1}^{2}}{2} \ln \frac{A_{a}^{2}}{A_{a}^{1}}+\frac{\lambda_{2}^{2}}{4} \ln \frac{A_{a}^{2}}{A_{a}^{1}}\right]^{-1} .
\end{aligned}
$$

A detailed analysis of Eq. (14) indicates that the worst-case scenario corresponds to the presence of discontinuities in $A_{a}$. Within the expected application scope of this new NRBC, such a case could be the simulation of the exhaust system of an ICE. According to Bermúdez et al. (2011), the maximum value along the whole cycle of the logarithm of the entropy level ratio at the exhaust pipe is 0.3 at $\mathrm{N}=2500 \mathrm{rpm}$. Considering this upper bound, Eq. (14) becomes:

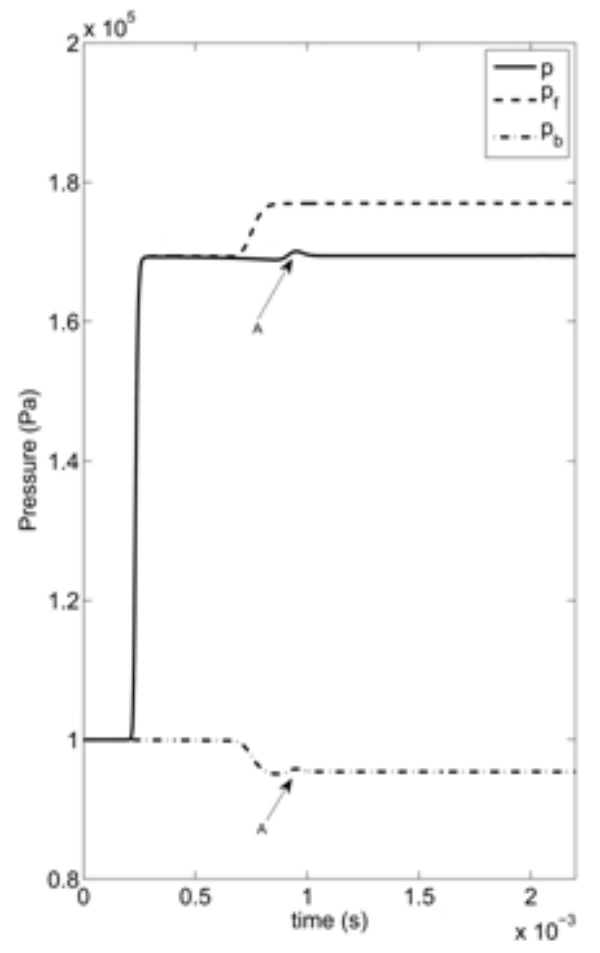

$$
\begin{aligned}
\frac{\beta_{2}^{2}-\tilde{\beta}_{2}^{2}}{\beta_{2}^{2}} & =\left[0.08\left(\lambda_{1}^{2}-\lambda_{1}^{1}\right)+0.15\left(a_{1}^{2}-a_{1}^{1}\right)\right] \\
& \times\left[\beta_{1}^{2}+\frac{a_{1}^{2}}{2} \ln \frac{A_{a}^{2}}{A_{a}^{1}}+\frac{\lambda_{2}^{2}}{4} \ln \frac{A_{a}^{2}}{A_{a}^{1}}\right]^{-1}
\end{aligned}
$$

showing that the importance of the error committed when assuming Eq. (12) is relatively small, since the corresponding differences are multiplied by coefficients much smaller than unity.

Therefore, it can be concluded that, for an anechoic case, the value of $\beta$ arriving at the boundary condition at the $N$-th time-step can be computed as:

$$
\begin{aligned}
\tilde{\beta}_{i n}^{N} & =\left[\beta_{i n}^{N-1}+\frac{a_{N-1}}{2} \ln \frac{A_{a}^{N}}{A_{a}^{N-1}}+\frac{\lambda_{N}}{4} \ln \frac{A_{a}^{N}}{A_{a}^{N-1}}\right] \\
& \times\left[1-\frac{1}{4} \ln \frac{A_{a}^{N}}{A_{a}^{N-1}}\right]^{-1} .
\end{aligned}
$$

In this way, an anechoic end can be modeled, thus removing the need for computing the flow in the downstream duct. One only needs to know $\lambda$ and $A_{a}$ arriving at the boundary at a given time $t$, the value of $\beta$ and $A_{a}$ which arrived at time $t-\Delta t$ and the speed of sound in the boundary at time $t-\Delta t$.

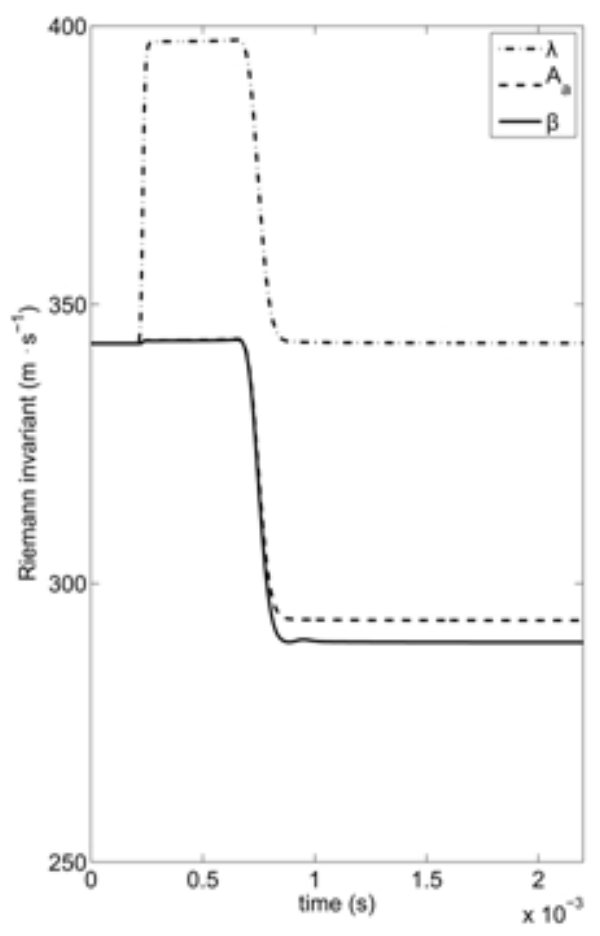

Fig. 7 Values of flow variables: pressure and pressure components (left) and Riemann variables (right) using new implementation of the NRBC. 
It is remarkable that the proposed approach is quite similar to that used in subsection 2.2, since in both cases the value of $\beta$ is varied only when there is a change in $A_{a}$. However, this variation is not directly $\Delta A_{a}$ but the expression given in Eq. (16). Moreover, stating that $\beta=A_{a}$ would also be incorrect when having pressure pulses superimposed on a mean flow, since at the initial time-step $\beta=a+u(\gamma-1) / 2 \neq a=A_{a}$, because $u$ is positive (considering that the reference pressure has been set equal to the initial pressure $\mathrm{p}_{0}=\mathrm{p}(\mathrm{t}=0)$, as discussed in Galindo et al. (2011)).

The validity of the new implementation was again checked by applying it to Sod's problem. The results obtained with the new NRBC are presented in Fig. 7. It may be seen that now the behavior is the same as for the ideal infinite duct, shown in Fig. 2. Again, some small disturbances appear when the discontinuity reaches the boundary (mark A), but these disturbances are damped and tend to disappear within a few timesteps.

\subsection{Incident pressure condition}

A similar treatment could be applied to an incident pressure boundary condition. However, in this case it is more difficult to find some nonhomentropic flow in which the problem could be solved in an independent way (i.e. not in the whole domain, but imposing boundary conditions from previous computations or experiments). A possible example might be a decoupled problem with a domain in which some regions are 1D elements. In such a situation, it would be possible to follow the same approach used for the anechoic end in order to reproduce the behavior of a nonreflecting incident pressure boundary condition in cases where changes in entropy are present.

\section{APPLICATION TO ANALYSIS OF ACOUSTIC RESPONSE OF MUFFLER}

In this section, a real application of the proposed NRBC is presented. The case chosen was the computation of the acoustic response of a 3D geometry similar to an exhaust muffler of an ICE. The results of the simulation were compared to experimental data obtained in an impulse test rig as described by Payri et al. (2000) and to the results obtained by using long inlet and outlet ducts to isolate the pressure signals (Broatch et al., 2005). The muffler analyzed (Fig. 8) was a rectangular parallelepiped with a volume of around 10 liters, which is adequate for the level of excitation imposed in the experiment. Inlet and outlet openings were located on the front and side surfaces, respectively, in order to capture the propagation of transversal modes (Munjal, 1987; Denia et al., 2001).

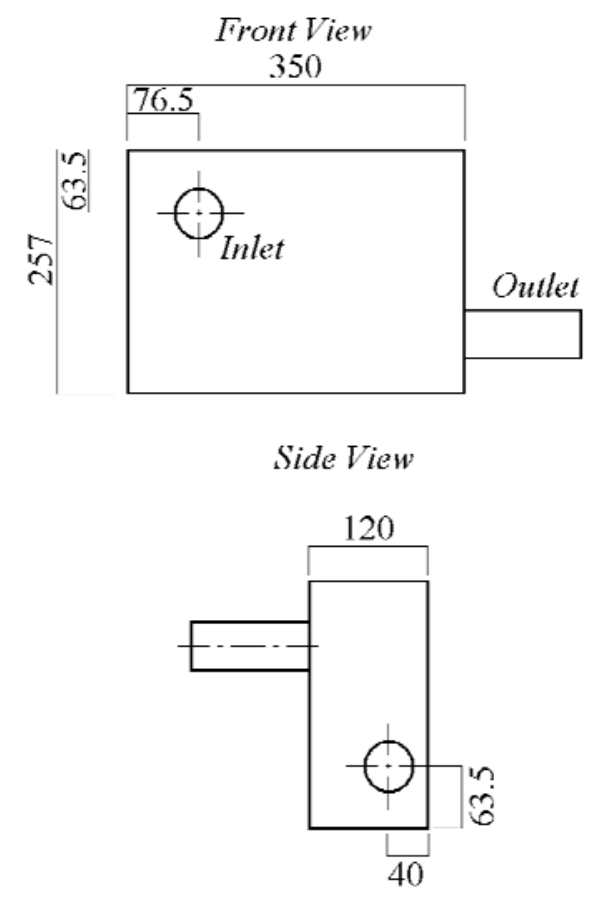

Fig. 8 Geometry of 3D muffler.

\subsection{Experimental facility}

In the experiments, the muffler is excited by means of a pressure pulse that provides a similar excitation at all the frequencies of interest. The incident pulse is generated by means of a solenoid valve that controls the discharge from a high pressure air tank (see Fig. 9). Three transducers are installed upstream and downstream of the muffler: transducer 1 records a reference pulse, while the pulse transmitted by the system is obtained with the third transducer. An additional test has to be carried out by removing the muffler from the set-up in order to obtain the isolated incident pressure pulse from the measurement recorded by transducer 2 . Once the incident and transmitted signals are obtained, the fast Fourier transform (FFT) is applied in order to compute different parameters such as the Transmission $\operatorname{Loss}(T L)$ of the system tested:

$T L(f)=20 \log \left|\frac{A(f)}{C(f)}\right|+10 \log \left|\frac{S_{u}}{S_{d}}\right|$ 
where $A(f)$ and $C(f)$ are the complex amplitudes of the incident and transmitted pulses, respectively, and $S_{u}$ and $S_{d}$ are the duct crosssections upstream and downstream of the muffler, respectively.

\subsection{Full three-dimensional simulation}

The commercial code ANSYS-FLUENT was used to perform the CFD calculations that simulate the experimental facility described above. ANSYS-FLUENT is based on a finite-volume discretization of the Navier-Stokes equations which, in this study, were solved with the socalled density-based coupled solver that uses fluxdifference splitting. For the time discretization a second order implicit scheme was used. The time step was set to $1 \times 10^{-5}$, which is smaller than the sampling frequency used in the test rig. The Realizable $k-\varepsilon$ turbulence model for high Reynolds numbers with wall function was chosen for closure.

The actual geometry and experimental conditions of the impulse test bench, described in the preceding section, were reproduced for the 3DCFD simulations. The computational domain was a structured hexahedral mesh consisting of the muffler $\left(1.6 \times 10^{5}\right.$ cells $)$ and the inlet and outlet ducts. The length of the inlet and outlet ducts (each $20 \mathrm{~m}, 0.6 \times 10^{5}$ cells) is the same as that used in the experimental facility in order to avoid reflections and to allow the isolated monitoring of the pulses. At the inlet, the pulse was represented by a temporal variation of the pressure at the inlet boundary condition. The outlet duct is needed to allow for the development of the flow before the pulse reaches the boundary. Since the temperature in the interior of the domain does not differ noticeably from the ambient temperature, the heat transfer through the wall was neglected.

The solution was monitored at the points corresponding to the locations of the transducers in order to obtain the response of the muffler. The methodology is essentially the same as in the experiments. Consequently, an additional simulation in which the muffler was replaced by a straight duct was performed in order to obtain the isolated incident pulse. Finally, the Transmission Loss $(T L)$ was calculated by applying Eq. (17). Broatch et al. (2005) proved that this methodology gives very accurate results when compared with the experiments in terms of $(T L)$, furthermore providing additional information on the $3 \mathrm{D}$ flow features in either the time or the frequency domains. Nevertheless, as previously stated, the computational cost of this methodology in real 3D geometry is high, which justifies the use of an NRBC to speed-up the calculation process.

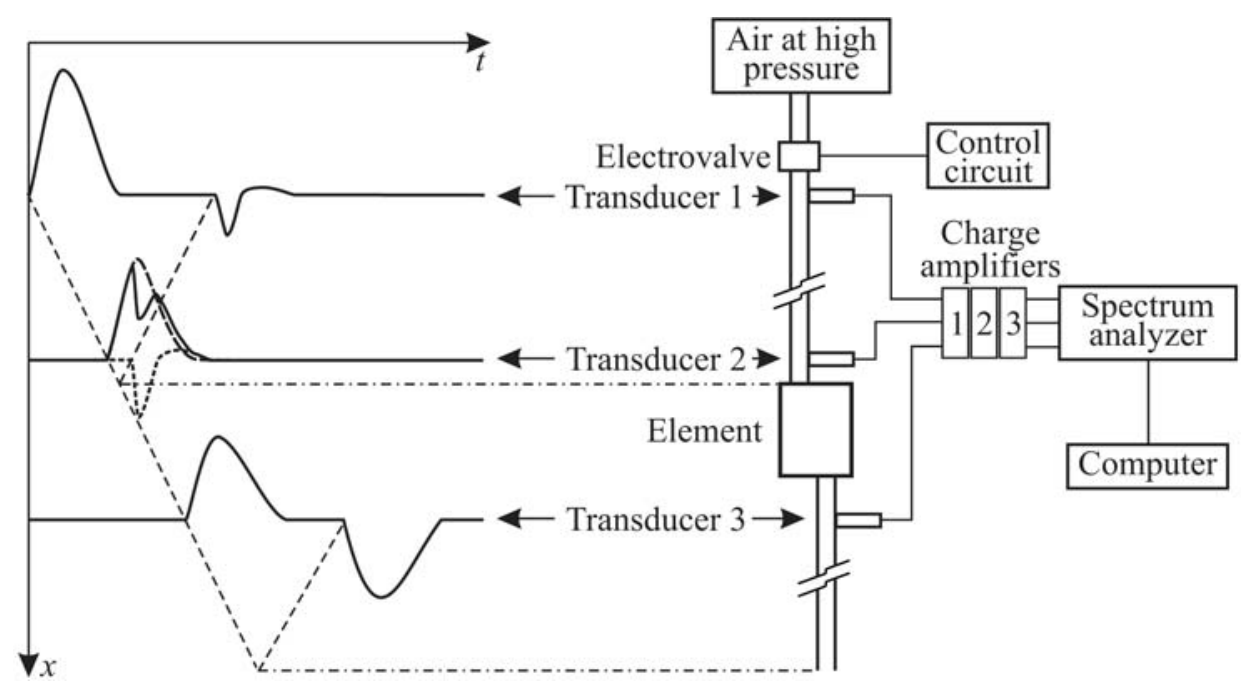

Fig. 9 Scheme of measurement setup.
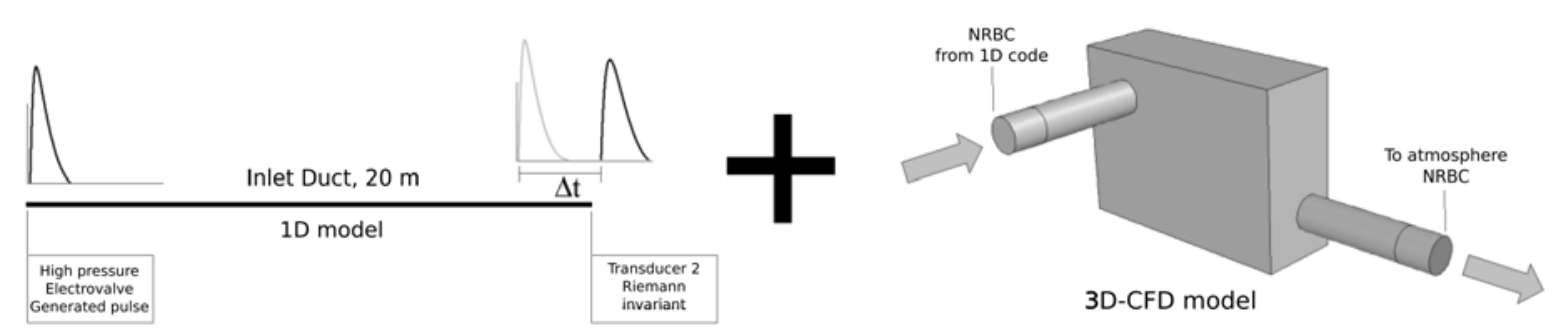

Fig. 10 Scheme of combined 1D-3D methodology. 


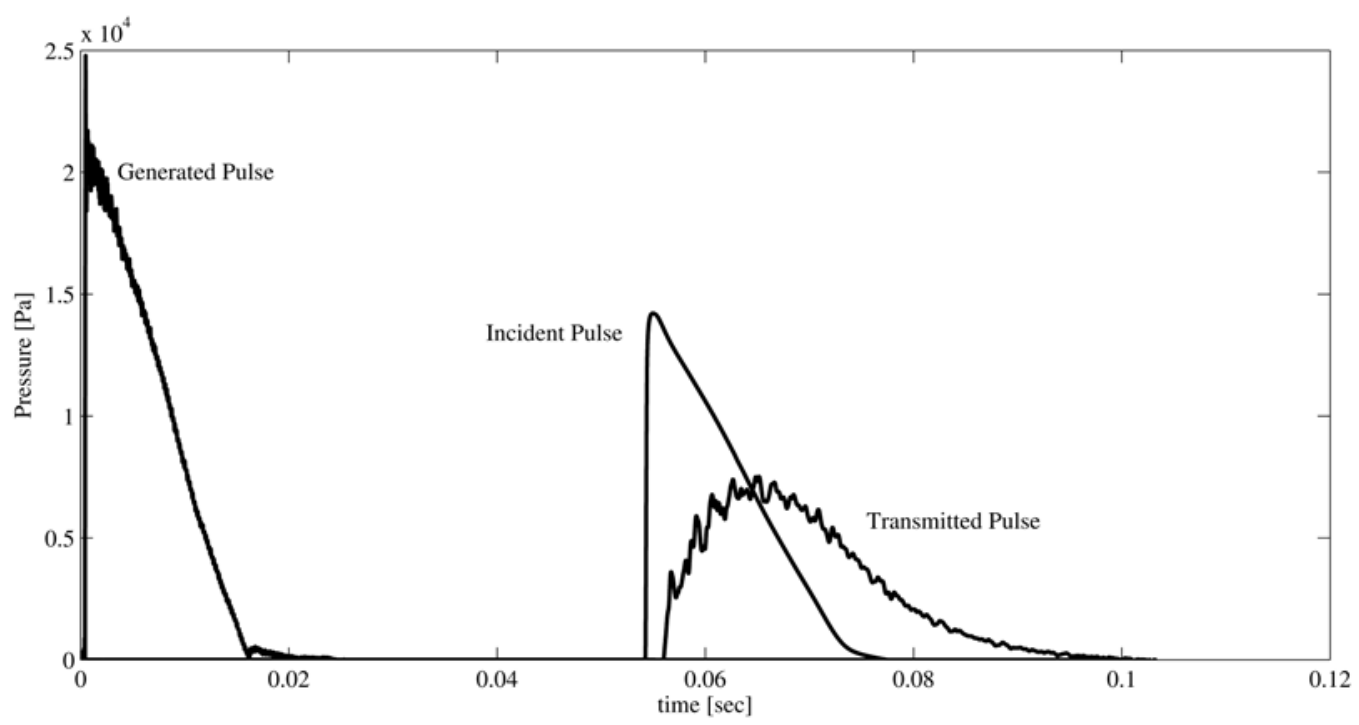

Fig. 11 Reference, incident and transmitted pulses obtained with full 3D CFD methodology.

\subsection{Proposed methodology}

As already mentioned, the aim of the proposed methodology, based on NRBCs, is to reduce the computational cost by avoiding the 3D flow computation of ducts. Since, in this particular case, the propagation of pressure waves in the inlet duct is not interesting, the problem was solved in a decoupled way, the inlet duct being calculated with a 1D CFD code: OpenWAM. This 1D-code computes the internal flow in pipes under the assumption of non-linear inviscid onedimensional flow, and has been extensively used (Galindo et al., 2009) to model transient compressible flows in simple geometries with very low computational requirements. The isolated incident pulse was therefore obtained with this simple, low computational cost model, and then incorporated into the 3D model by means of an incident pressure NRBC. Additionally, the anechoic end presented in this paper was used at the outlet duct, so that a long duct was not required. Other parameters defining the three-dimensional model such as mesh, turbulence or discretization schemes among others were the same as those chosen for the full three-dimensional calculations.

As indicated in section 2, the reduction in CPUtime with respect to the full $3 \mathrm{D}$ computation is due to two different effects. CPU-time per timestep is reduced in this case by around 35\% which is fairly close to the corresponding decrease in the number of cells. Of course, the importance of this reduction depends on the number of mesh elements corresponding to the system under study. The second source of CPUtime reduction comes from the reduction in the total simulated time achieved when pulse propagation along the ducts is not computed. Fig. 11 shows the temporal evolution of the pulses along the system. The reference pulse needs about 0.06 seconds to arrive at the second transducer, where the incident pulse is monitored. During this time the system under study does not receive any pressure excitation, so that no useful information is extracted from the computation in this period, which amounts to about half of the duration of the full 3D computation. Consequently, the CPU-time consumed will decrease down to values close to $50 \%$. Therefore, the overall reduction in computation time due to the combination of both effects in the present case was about $67.5 \%$. This reduction depends on the pulse duration, and on the characteristic length of the ducts and the element being tested.

Finally, comparison of the experimental Transmission Loss and the results obtained with both numerical methods (full 3D and combined 1D-3D with NRBCs) is presented in Fig. 12. Good agreement between measurement and calculations can be observed. Both calculation methods accurately predict the peaks and the pass-band frequencies. The results obtained with the full 3D and the combined 1D-3D simulations are quite similar (less than $1 \mathrm{~dB}$ of difference) except in the maximum attenuation of the first narrow peak, in which the error is about $3 \mathrm{~dB}$. It is worth mentioning that recovering the exact value of a peak in an acoustic analysis is not as important as capturing the general behavior. In fact such peaks are not relevant when the muffler is subject to a broadband excitation, which is the most usual case in practice. Additionally, this error is justified in the current application when the savings in CPU-time are considered. 


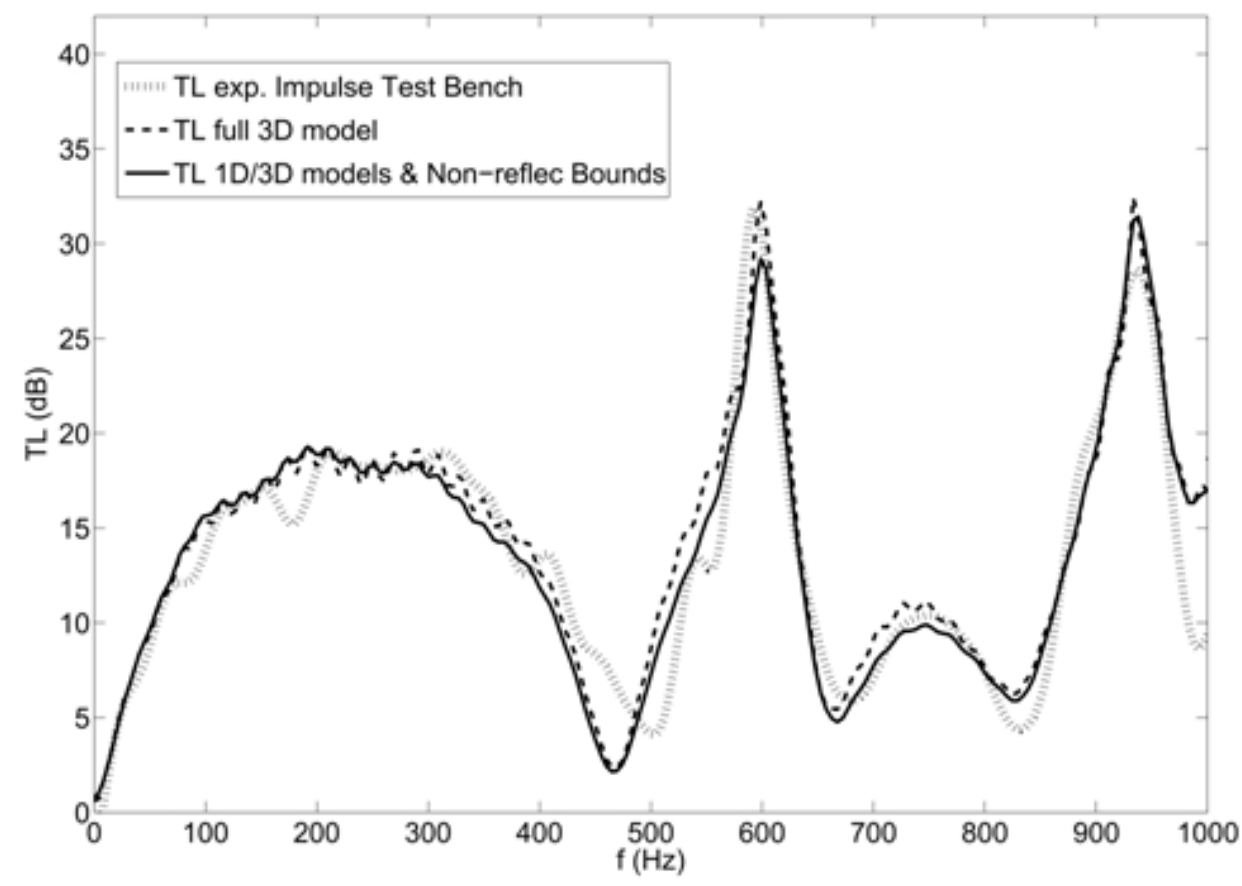

Fig. 12 Comparison of measured transmission loss with those from proposed methodology and full 3D model.

\section{SUMMARY AND CONCLUSIONS}

In this paper, both a methodology to implement NRBCs in a 3D CFD commercial code (such as ANSYS-FLUENT) for its application to internal flow simulations, and the development of a new NRBC, were presented. The transfer of information across the boundary was accounted for by means of the MoC. One of the key points solved in the implementation of a boundary condition in a commercial code was the acquisition of the values of the flow variables in the domain. The performance of the new proposal was compared with a former definition proposed by the authors, and also with the NRBCs available in ANSYS-FLUENT, through their application to Sod's shock tube. Comparison of the solution obtained for an ideal infinite tube with the results obtained with the different nonreflecting boundary conditions analyzed, indicated that the non-reflecting boundary conditions available in ANSYS-FLUENT show some spurious reflections, especially when dealing with changes in entropy level. The proposed formulation showed improved performance with respect to the former definition and also to ANSYS-FLUENT built-in boundary conditions. In this paper, only the particular case of an anechoic end was addressed, however, the same conclusions might be extracted for an incident pressure boundary condition.

Finally, a realistic application to the determination of the acoustic behavior of a $3 \mathrm{D}$ muffler was considered, comparing the results obtained with those of experimental tests and a full 3D CFD computation. The comparison indicated that the results in both the time and the frequency domains are very similar to those obtained with the full 3D-CFD simulation. Moreover, the computational cost is reduced by a factor of $3(67.5 \%)$ when simulating an NRBC in the way presented on this paper. This suggests that the proposed methodology might constitute a suitable tool for design and evaluation purposes.

\section{ACKNOWLEDGEMENT}

This work has been partially supported by Ministerio de Ciencia e Innovación through grant No. DPI2009-14290. The authors wish to thank Dr. David R. Perry for his kind assistance in manuscript editing.

\section{REFERENCES}

1. ANSYS Inc. (2009). Ansys Fluent 12.0 User's Guide. Canonsburg, PA: ANSYS Inc.

2. Atassi OV (2004). Nonreflecting boundary conditions for the time-dependent convective wave equation in a duct. Journal of Computational Physics 197(2):737-758.

3. Benson RS (1982). The Thermodynamics and Gas Dynamics of Internal Combustion Engines. Volume 1, Oxford: Oxford University Press. 
4. Benson RS, Garg RD, Woollatt D (1964). A numerical solution of unsteady flow problems. International Journal of Mechanical Sciences 6(1):117-144.

5. Bermúdez V, Serrano JR, Piqueras P, GarcíaAlfonso O (2011). Assessment by means of gas dynamic modelling of a pre-turbo diesel particulate filter configuration in a turbocharged HSDI diesel engine under fullload transient operation. Proceedings of the Institution of Mechanical Engineers Part D Journal of Automobile Engineering 225(9):1134-1155.

6. Bogey C, Bailly C (2002) Three-dimensional non-reflective boundary conditions for acoustic simulations: far field formulation and validation test cases. Acta Acustica united with Acustica 88(4):463-471.

7. Broatch A, Margot X, Gil A, Denia FD (2005). A CFD approach to the computation of the acoustic response of exhaust mufflers. Journal of Computational Acoustics 13(2):301-316.

8. Dea JR, Giraldo FX, Neta B (2009). Highorder non-reflecting boundary conditions for the linearized 2-D Euler equations: No mean flow case. Wave Motion 46(3):210-220.

9. Denia FD, Albelda J, Fuenmayor FJ, Torregrosa AJ (2001). Acoustic behaviour of elliptical chamber mufflers. Journal of Sound and Vibration 241(3):401-421.

10. Dorodnicyn LW (2010). Artificial boundary conditions for high-accuracy aeroacoustic algorithms. SIAM Journal on Scientific Computing 32(4):1950-1979.

11. Elfaghi AM, Asrar W, Omar AA (2010). Higher order compact-flowfield dependent variation (HOC-FDV) solution of onedimensional problems. Engineering Applications of Computational Fluid Mechanics 4(3):434-440.

12. Engquist B, Majda A (1977). Absorbing boundary conditions for the numerical evaluation of waves. Mathematics of Computation 31(139):629-651.

13. Galindo J, Tiseira A, Fajardo P, Navarro R (2011). Coupling methodology of 1D finite difference and 3D finite volume CFD codes based on the Method of Characteristics. Mathematical and Computer Modelling 54(78):1738-1746.

14. Galindo J, Serrano JR, Arnau FJ, Piqueras P (2009). Description of a semi-independent time discretization methodology for a onedimensional gas dynamics model. Journal of
Engineering for Gas Turbines and PowerTransactions of the ASME 131(3):034504.

15. Givoli D (2004). High-order local nonreflecting boundary conditions: a review. Wave Motion 39(4):319-326.

16. Hagstrom T, Hariharan SI (1988). Accurate boundary conditions for exterior problems in gas dynamics. Mathematics of Computation 51(184):581-597.

17. Lindquist JM, Neta B, Giraldo FX (2010). A spectral element solution of the Klein-Gordon equation with high-order treatment of time and nonreflecting boundary. Wave Motion 47(5):289-298.

18. Liu Q, Vasilyev OV (2010). Nonreflecting boundary conditions based on nonlinear multidimensional characteristics. International Journal for Numerical Methods in Fluids 62(1):24-55.

19. Luján JM, Bermúdez V, Serrano JR, Cervelló C (2002). Test bench for turbocharger groups characterization. SAE Paper 2002-01-0163.

20. Montenegro G, Onorati A (2009). Modeling of silencers for IC engine intake and exhaust systems by means of an integrated 1D-multiD approach. SAE International Journal of Engines 1(1):466-479.

21. Moríñigo JA, Salvá JJ (2010). Robust nonreflecting boundary conditions for the simulation of rocket nozzle flow. Aerospace Science and Technology 14(6):429-441.

22. Mucklow GF, Wilson AJ (1955). The attenuation and reflection of compression waves propagated in pipes. Proceedings of the Institution of Mechanical Engineers 169:69-80.

23. Munjal ML (1987). Acoustics of Ducts and Mufflers. New York: Willey.

24. Onorati A, Montenegro G, D’Errico G (2006). Prediction of the attenuation characteristics of IC engine silencers by 1-D and multi-D simulation models. SAE Paper 2006-01-1541.

25. OpenWAM. URL: http://www.OpenWAM. org.

26. Patil AR, Sajanpawar PR, Masurekar VV (1996) Acoustic three dimensional finite element analysis of a muffler. SAE Paper 960189.

27. Payri F, Desantes JM, Broatch A (2000). Modified impulse method for the measurement of the frequency response of acoustic filters to weakly nonlinear transient excitations. Journal of the Acoustical Society of America 107(2):731-738.

28. Payri F, Desantes JM, Torregrosa AJ (1995). Acoustic boundary condition for unsteady 
one-dimensional flow calculations. Journal of Sound and Vibration 188(1):85-110.

29. Poinsot TJ, Lele SK (1992). Boundary conditions for direct simulations of compressible viscous flows. Journal of Computational Physics 101(1):104-129.

30. Qamar A, Hasan N, Sanghi S (2010). A new spatial discretization strategy of the convective flux term for the hyperbolic conservation laws. Engineering Applications of Computational Fluid Mechanics 4(4):593611.

31. Selle L, Nicoud F, Poinsot T (2004). Actual impedance of non-reflecting boundary conditions: implications for computation of resonators. AIAA Journal 42(5):958-964.

32. Sod GA (1978). A survey of several finite difference methods for systems of nonlinear hyperbolic conservation laws. Journal of Computational Physics 27(1):1-31.

33. Tam CKW (1995). Computational aeroacoustics: issues and methods. AIAA Journal 33(10): 1788-1796.

34. Thompson KW (1987). Time-dependent boundary conditions for hyperbolic systems. Journal of Computational Physics 68(1):1-24.

35. Thompson KW (1990). Time-dependent boundary conditions for hyperbolic systems, II. Journal of Computational Physics 89(2):439-461.

36. Tsuji T, Tsuchiya T, Kagawa Y (2002). Finite element and boundary element modelling for the acoustic wave transmission in mean flow medium. Journal of Sound and Vibration 255(5):849-866.

37. Wang B, Xu H (2007). A method based on Riemann problem in tracking multimaterial interface on unstructured moving grids. Engineering Applications of Computational Fluid Mechanics 1(4):325-336. 